\title{
Inverted Solution Processable OLEDs Using a Metal Oxide as an Electron Injection Contact ${ }^{1}$
}

\author{
By Henk J. Bolink,** Eugenio Coronado, Diego Repetto, Michele Sessolo, Eva M. Barea, Juan Bisquert, \\ Germà Garcia-Belmonte, Jan Prochazka and Ladislav Kavan
}

\begin{abstract}
A new type of bottom-emission electroluminescent device is described in which a metal oxide is used as the electron-injecting contact. The preparation of such a device is simple. It consists of the deposition of a thin layer of a metal oxide on top of an indium tin oxide covered glass substrate, followed by the solution processing of the light-emitting layer and subsequently the deposition of a high-workfunction (air-stable) metal anode. This architecture allows for a low-cost electroluminescent device because no rigorous encapsulation is required. Electroluminescence with a high brightness reaching $5700 \mathrm{~cd} \mathrm{~m}-2$ is observed at voltages as low as $8 \mathrm{~V}$, demonstrating the potential of this new approach to organic light-emitting diode (OLED) devices. Unfortunately the device efficiency is rather low because of the high current density flowing through the device. We show that the device only operates after the insertion of an additional hole-injection layer in between the light-emitting polymer (LEP) and the metal anode. A simple model that explains the experimental results and provides avenues for further optimization of these devices is described. It is based on the idea that the barrier for electron injection is lowered by the formation of a space-charge field over the metal-oxide-LEP interface due to the build up of holes in the LEP layer close to this interface.
\end{abstract}

\section{Introduction}

Interest in organic light-emitting diodes (OLEDs) for use in display and lighting applications is increasing. This is mainly because of reports of new breakthroughs in device efficiencies, lifetimes, and achievable colors, including white. ${ }^{1}$ However, multilayer devices are needed to obtain these high performance levels and, additionally, the devices need to be rigorously encapsulated because of the reactive cathodes or electron injection layers that are used. To enter successfully the general lighting market, OLEDs require a strong reduction in the cost of the devices as well as high performance levels. In this respect, it is of particular importance to be able to generate electroluminescence from devices using air-stable charge-injection interfaces. Although some examples exist, these devices rely on the presence of ionic charges to generate a dipole across the metal-light-emitting-layer interface and their reported lifetimes are low. ${ }^{2}-{ }^{4}$ Metal oxides are, in principle, promising candidates that may lead to good charge injection as they combine properties such as high transparency, low resistance, and air stability. Recently, there have been reports about the use of metal oxides as charge injection layers. They range from ultrathin layers on the anode side to nanostructured layers on the cathode side of the devices. ${ }^{5}-{ }^{6}{ }^{7}{ }^{8}-{ }^{9}$ The use of a hole-blocking metaloxide material on the anode side modifies the device efficiency by adjusting the charge balance in the device ${ }^{6}$. A more beneficial use of the metal-oxide layer is as an alternative cathode material. The use of an unreactive metal oxide as the cathode is appealing as this would allow the

\footnotetext{
${ }^{1}$ This work has been supported by the European Union (HETEROMOLMAT, STRP 516982) the Spanish Ministry of Education and Science (MEC) (MAT2007-61584, CSD2007-00010 and MAT2004-05168) the Czech Ministry of Education (grant no. LC510), and the Generalitat Valenciana. H.J.B. acknowledges the support of the Program "Ramon y Cajal" of the MEC.

${ }^{* *}$ Dr. H. J. Bolink, Prof. E. Coronado, Dr. D. Repetto, M. Sessolo Instituto de Ciencia Molecular, Universidad de Valencia PO Box 22085, E-46071 Valencia, (Spain) E-mail: henk.bolink@uv.es

Dr. E. M. Barea, Prof. J. Bisquert, Dr. G. Garcia-Belmonte Departament de Física, Universitat Jaume I 12071 Castelló, (Spain)

J. Prochazka, Prof. L. Kavan

J. Heyrovský Institute of Physical Chemistry, v.v.i., Academy of Sciences of the Czech Republic 18223 Prague 8 (Czech Republic)
} 
preparation of OLEDs requiring no, or only simple, encapsulation. This would significantly reduce costs and, therefore, increase the feasibility of the use of OLEDs in display and especially lighting applications.

Recently, Morii et. al. showed that it is possible to generate electroluminescence from a device that uses $\mathrm{TiO}_{2}$ as the cathode ${ }^{7}$. They reached brightness levels of $700 \mathrm{~cd} \mathrm{~m}^{-2}$ at a driving voltage of $6 \mathrm{~V}$. The current to light efficiency, however, was of the order of $0.1 \mathrm{~cd} \mathrm{~A}^{-1}$ because of the high current densities flowing through the device.

The mechanism of operation of the OLEDs using a simple undoped metal oxide such as titanium dioxide as the cathode is intriguing. An energetic barrier exists for the injection of electrons from the conduction band of the metal oxide to the lowest unoccupied molecular orbital (LUMO) of the light-emitting material (Fig. 1). LUMO energies for most light-emitting polymers (LEPs), as well as for electron transporting materials used in multilayer OLEDs, range from -2.5 to $-3.0 \mathrm{eV}$. This mismatch between the conduction band of the metal oxide and the LUMO levels in the organic electron transport moieties, in principle, would inhibit efficient device operation. Nevertheless, when making use of the LEP, poly(9,9dioctylfluorene-cobenzothiadiazole) (F8BT), electroluminescence was observed. F8BT has a low LUMO level that decreases the energy gap between the metal oxide and the LUMO of the LEP as much as possible. The principal objective of this paper is to shed light on the operational mechanism of inverted metal-oxide OLEDs, hereafter called HyLEDs (Hybrid OLEDs). Therefore, we have focused our work on the same LEP, F8BT. As well as having a low LUMO energy level, F8BT is one of the few examples of a predominantly electron transporting LEP, which may enhance the operation of this inverted OLED devices that uses a metal oxide as the cathode ${ }^{10}$. In this work, we comment on the use of an inverted OLED structure using a dense titanium dioxide layer as the electron-injection contact. We have been able to obtain very bright green-light-emitting electroluminescent devices that reach luminance values as high as $2500 \mathrm{~cd} \mathrm{~m}^{-2}$ at voltages as low as $6 \mathrm{~V}$. Such high brightness devices could only be obtained using F8BT as the LEP. However, the current density accompanying these high brightness levels is also high, resulting in low current efficiency values around $0.1 \mathrm{~cd} \mathrm{~A}^{-1}$. In this paper, we present evidence for the mechanism of device operation, which is dominated by hole currents, and present avenues for further device optimization. The paper is structured as follows: first we comment on the preparation and properties of the $\mathrm{TiO}_{2}$ layer, followed by the analysis of sandwiched F8BT devices between the $\mathrm{TiO}_{2}$ and $\mathrm{Au}$ electrodes. Subsequently, we change the device architecture by adding a hole injection layer in between the F8BT and the $\mathrm{Au}$, which results in a dramatic increase in current density and light output. From this observation it is deduced that the current is predominantly a hole current. Additionally, we attempt to increase the device performances by changing the charge distribution in the device by adding hole traps. The presence of the hole traps reduces significantly the current density in the device, which corroborates the model of a hole dominated current. Additionally, it appears that the electron injection in these devices is dependent on the density of holes that accumulate at the electron injection interface. Finally, we show the extreme case, in a device without the $\mathrm{TiO}_{2}$ layer. Here, the indium tin oxide (ITO) on the glass substrate functions as the cathode contact. In this device architecture, the barrier for electron injection is close to $1.2 \mathrm{eV}$. However, electron injection can occur because of the build up of holes at the ITO/F8BT interface. In such a device layout we observed electroluminescence reaching brightness levels of $200 \mathrm{~cd} \mathrm{~m}^{-2}$ at $8 \mathrm{~V}$.

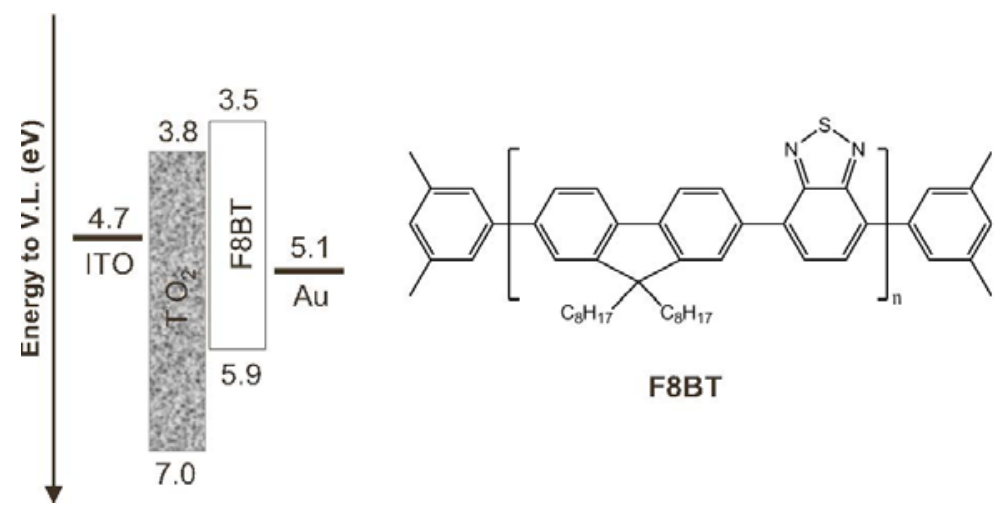

Figure 1. Scheme of the energy levels of the materials involved in the inverted metal-OLEDs use thin layers of organic materials that oxide OLED and chemical structure of the light-emitting polymer F8BT. 


\section{Results and Discussion}

OLEDs use thin layers of organic materials that function as the hole and electron transporters. Because of the low charge mobilities in these materials, the organic layers are required to be thinner than $100 \mathrm{~nm}$. Therefore, it is very important, for a proper device operation, to have very well defined (flat) substrates to deposit the organic layers onto. This is important since after the deposition of the thin LEP layer there should not be any direct contact between $\mathrm{TiO}_{2}$ and the counter electrode. In this study, we used spray pyrolysis, which is a known technique that is useful for the preparation of transparent and homogenous titanium dioxide films. ${ }^{11}$

The morphology of the metal-oxide layer has therefore been investigated by atomic force microscopy (AFM). The image displayed in Figure 2 shows that the surface of the $\mathrm{TiO}_{2}$ layer was homogeneous and flat, with a very low roughness (root mean square deviation, $\mathrm{rms}=2.6 \mathrm{~nm}$ ) that was mainly dependent on the ITO-covered-glass substrate morphology used for film deposition ( $\mathrm{rms} \sim 3 \mathrm{~nm}$ ). Therefore, we can reasonably assume that the oxide film was fully covered by the LEP layer ( $>50 \mathrm{~nm}$ thickness). ${ }^{12}$

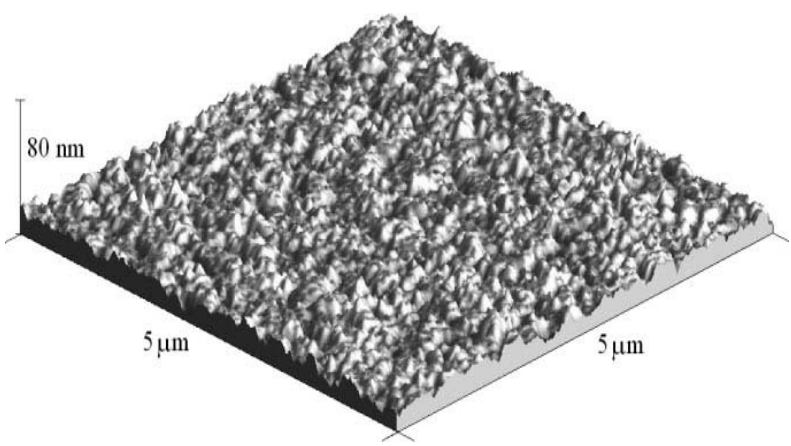

Figure 2. 3D topography of a titanium oxide layer on an ITO/glass sub-strate.

Apart from the flatness and the thickness of the $\mathrm{TiO}_{2}$, it is important to ensure there are no leakage paths to the ITO. The thickness of the films used for the preparation was estimated by scanning electron microscopy (SEM), which analyzed the cross section of a $\mathrm{TiO}_{2}$-coated and fractured sample (Fig. 3). In the image, the two interfaces between the glass, ITO, and metal oxide, are clearly visible and the thickness of the titanium oxide layer is approximately $80 \mathrm{~nm}$. Additionally, this image shows that the $\mathrm{TiO}_{2}$ is densely packed and therefore completely shields the underlying ITO electrode from contact with the F8BT in the HyLED device.

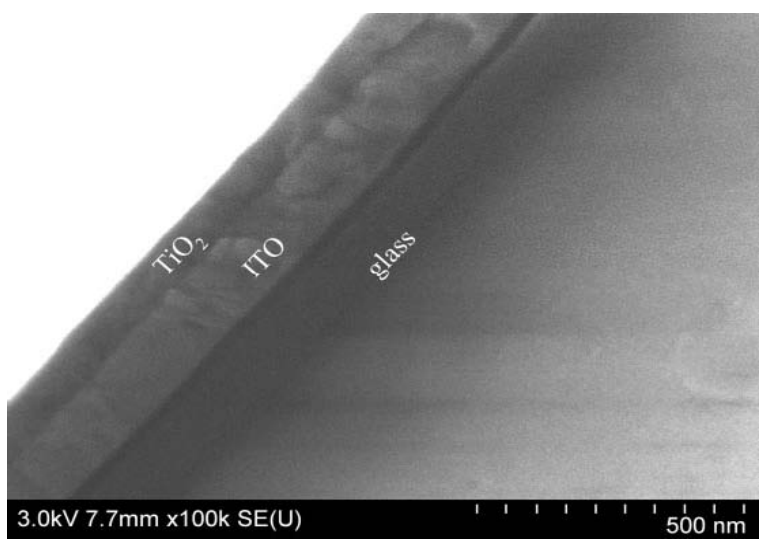

Figure 3. SEM cross-section image of a $\mathrm{TiO}_{2} / \mathrm{ITO} /$ glass fractured sample 
The current density versus voltage for an $\mathrm{ITO} / \mathrm{TiO}_{2} / \mathrm{F} 8 \mathrm{BT} / \mathrm{Au}$ device is depicted in Figure 4. The experiment was carried out with forward and reverse bias, biasing the gold electrode to positive and negative, respectively. It was immediately obvious that there was only a small difference between the current densities when the device was biased in forward or reverse mode. In both cases the current density increased after a turn-on voltage (approximately $2 \mathrm{~V}$ in reverse bias and $3 \mathrm{~V}$ in forward bias). The offset of the current density at $0 \mathrm{~V}$ is not completely understood but may be due to charge accumulation at the metal-oxide-organic interface after the initial voltage ramp. However, in both bias directions the current density is very low, which means that no significant injection of carriers was achieved. This was not completely unexpected as the energy difference for charge injection is large (Fig. 1). In the forward bias direction (Au positive and ITO negative), the barrier for hole injection is $0.8 \mathrm{eVand}$ the barrier for electron injection is around $0.9 \mathrm{eV}$. In the reverse bias direction (ITO positive and Au negative), the injection barriers are even larger ( $2.3 \mathrm{eV}$ for holes and $1.6 \mathrm{eV}$ for electrons). Therefore, the current flow observed in this simple device is likely to be caused by imperfections in the device structure that cause a leakage current. Thus, to reach the objective of electron emission, at least one of the interface barriers needs to be decreased. In a first approach we focused on the top contact, the Au anode layer. To decrease the barrier between the Au and the F8BT, a hole injection layer can be employed. It has been shown that the use of transition metal oxides (TMO) can greatly lower the injection barrier for hole injection. ${ }^{13}{ }^{14}$ The TMO functions as the effective charge-injection layer. It is assumed to form a chargetransfer complex with organic materials, increasing the local carrier density and hence forming an Ohmic contact. ${ }^{15}$ Therefore we introduced a thin $\mathrm{MoO}_{3}$ hole-injection layer, using high-vacuum thermal evaporation, between the light-emitting polymer (LEP) and the gold anode.

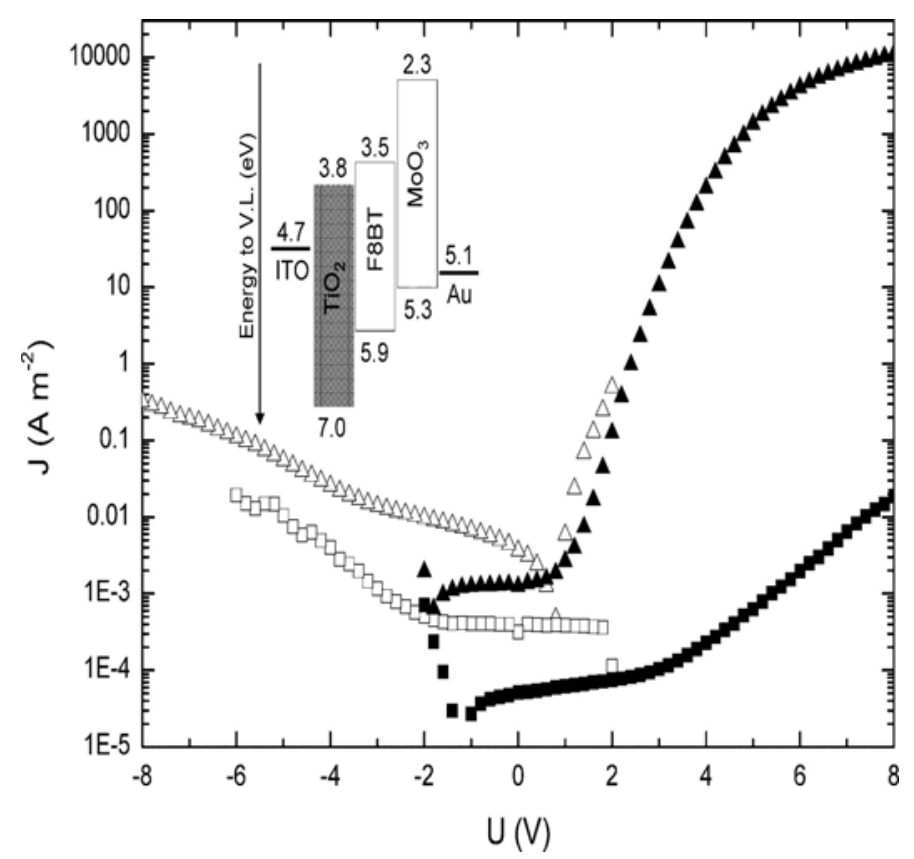

Figure 4. Current density versus applied bias for anITO/TiO $/ 2 / F 8 B T / A u$ (squares) and an $\mathrm{ITO} / \mathrm{TiO}_{2} / \mathrm{F} 8 \mathrm{BT} / \mathrm{MoO} / \mathrm{Au}($ triangles) device in forward $(\mathrm{Au}=$ positive) ( fullsymbols) and reverse bias ( $\mathrm{Au}=$ negative) ( open symbols). Inset shows a schematic presentation of the device layout with the main energy levels.

The incorporation of this thin $(20 \mathrm{~nm})$ layer of $\mathrm{MoO}_{3}$ between the F8BT and the Au anode resulted in a tremendous increase in current density under forward bias, reaching levels close to $10000 \mathrm{~A} \mathrm{~m}^{-2}$ at $8 \mathrm{~V}$. This seems to demonstrate that the incorporation of the $\mathrm{MoO}_{3}$ layer greatly enhances the injection of holes into the F8BT layer. The low current density under reverse bias indicates that neither holes (from the $\mathrm{TiO}_{2}$ layer) nor electrons (from the $\mathrm{MoO}_{3} / \mathrm{Au}$ interface) are efficiently injected into the F8BT layer. The latter observation is important as $\mathrm{MoO}_{3}$ has also been used as a recombination layer in tandem-cell light-emitting diodes, in which it acted as an electron and hole-injection contact. ${ }^{15}$ It seems, however, that in our configuration the $\mathrm{MoO}_{3}$ is not capable of injecting electrons into the F8BT layer.

The device with the $\mathrm{MoO}_{3}$ layer emitted light with a high brightness, reaching $5700 \mathrm{~cd} \mathrm{~m}^{-2}$ at $8 \mathrm{~V}$. The turn-on voltage was as low as $2.4 \mathrm{~V}$, which indicates that the barrier for electron injection is low. To be able to compare these results with the F8BT grade used in our HyLED configuration, we prepared a 
standard OLED device with F8BT as the active layer. Here, we used PEDOT:PSS (poly(ethylene dioxythiophene): poly(styrene sulfonate)) as the hole-injection layer and $\mathrm{Ba} / \mathrm{Ag}$ as the cathode. Figure 5 shows that the light output of the HyLED device was higher than that of the standard OLED devices, (at 8 $\mathrm{V}$, the HyLED device had almost five times higher brightness levels).

The efficacy (current to light efficiency) of the HyLED device, however, was somewhat lower than the standard device (Fig. 6). The difference in efficacy may be related to a difference in outcoupling as the HyLED device had negative bias on the ITO, whereas the ITO in the normal OLED configuration was positively biased. This means that the recombination zone could be at opposing interfaces, which may result in a decrease in outcoupling efficiency. It is beyond the scope of this paper to determine the outcoupling efficiency.

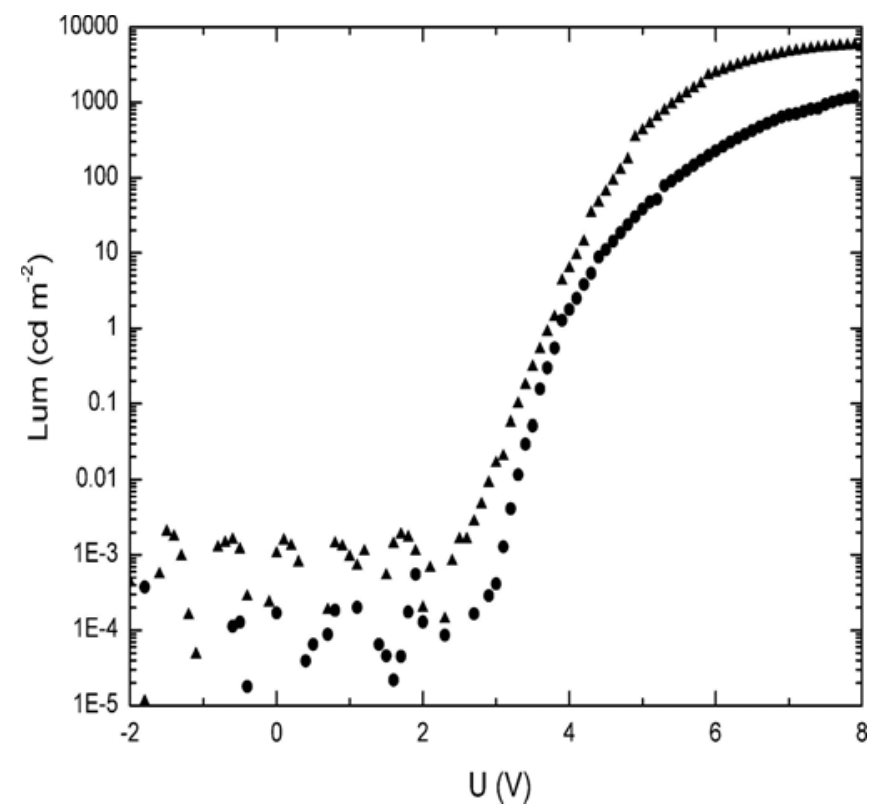

Figure 5. Luminance versus driving voltage for an ITO/TiO2/F8BT/MoO3/Au device in which the ITO is biased negatively ( triangles) and for a ITO/PEDOT/F8BT/Ba/Ag standard device in which the ITO is biased positively ( circles).

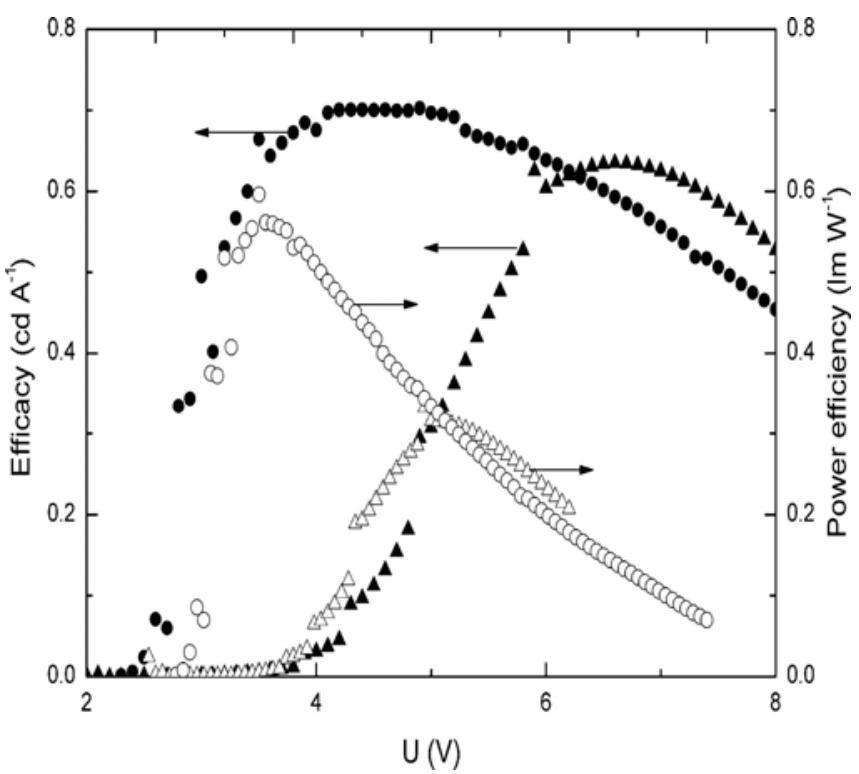

Figure 6. Efficacy ( fullsymbols) and powe refficiency ( open symbols) ver-sus driving voltage for $\mathrm{ITO} / \mathrm{TiO}_{2} / \mathrm{F} 8 \mathrm{BT} / \mathrm{MoO}_{3} / \mathrm{Au}$ ( triangles) in which the ITO is biased negatively and for a standard device ITO/PEDOT/F8BT/Ba/Ag ( circles) in which the ITO is 
As F8BT is reported to have a higher electron mobility than hole mobility, ${ }^{16}$ in a first approximation one would assume that electrons are the primary carriers that travel rapidly across the thin film and get blocked by the $\mathrm{MoO}_{3}$, where they recombine with the injected holes. Such a mechanism for an F8BTbased OLED using different cathode materials was described by Murata et. al. ${ }^{17}$ They showed that the current in a hole-only device (using gold as the cathode) is injection-limited. Upon applying a lowerworkfunction metal as the cathode a strong increase in current density was observed. The authors attributed this increase in current density to electron-assisted hole injection. The accumulation of electrons close to the F8BT/anode interface creates an interfacial field that allows for the injection of holes into the F8BT.

It does not seem likely that the electrons accumulate at the $\mathrm{F} 8 \mathrm{BT} / \mathrm{MoO}_{3}$ interface in the HyLED configuration for two reasons. Firstly, the electron-injection interface is not altered and the results obtained for devices without $\mathrm{MoO}_{3}$ indicate that no current was flowing, which suggests that the $\mathrm{TiO}_{2} /$ F8BT is a blocking contact. Secondly, the hole-injection barrier is significantly lowered by the insertion of the $\mathrm{MoO}_{3}$ layer, which is why we will discuss the case of the other extreme, in which the first carriers to be injected are the holes. Although there is a significant gap between the workfunction of gold and the highest occupied molecular orbital (HOMO) of the F8BT, the additional $\mathrm{MoO}_{3}$ charge-injection layer is capable of decreasing the barrier for hole injection such that an Ohmic contact is generated. This facilitates the efficient injection of holes into the F8BT layer. If the holes are indeed the primary carriers entering the F8BT layer, they will be transported towards the $\mathrm{TiO}_{2}$ counter electrode under the influence of the external electric field. $\mathrm{As} \mathrm{TiO}_{2}$ is an n-type semiconductor it can block, at least partially, the incoming holes, which subsequently creates a strong space-charge field across the $\mathrm{TiO}_{2} / \mathrm{F} 8 \mathrm{BT}$ interface. A possible layout of the modification of the barriers is shown in Figure 7.

(a)

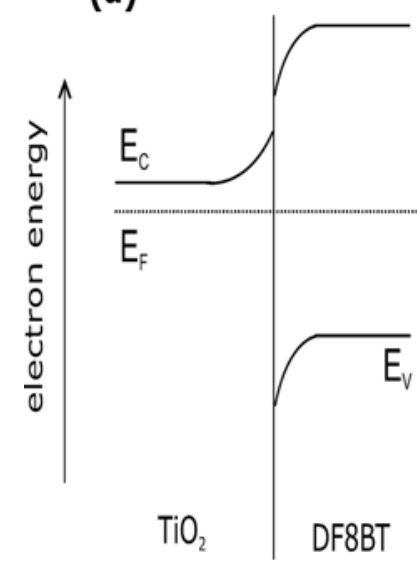

(b)

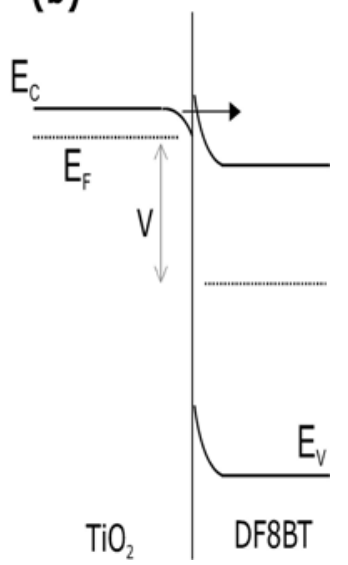

Figure 7. Energy diagram of the TiO2/F8BT interface. $E \mathrm{c}$ and $E \mathrm{~V}$ are the energies of the conduction and valence band, respectively, $E F$ is the Fermi level, and $V$ is the bias voltage. a) In equilibrium the two materials align their Fermi levels. The $n$-TiO2 side shows a depletion layer, while the F8BT shows an electron-rich layer at the interface. b) Under forward bias, electrons accumulate at the $\mathrm{TiO} 2$ side and holes at the F8BT side of the interface.

Under forward bias, the $\mathrm{TiO}_{2}$ obtains an electron rich accumulation layer, while holes accumulate at the F8BT side of the barrier. This accumulation layer can be quite thin, so tunneling of electrons across the interface becomes possible.

It is thus conceivable that the electrons are only injected over the energetic barrier of $0.7 \mathrm{eV}$ into the F8BT after the build up of this interfacial field. When they are, they rapidly recombined with the large concentration of holes that built up in the F8BT. This decreases the space charge limitation for the hole current and as a result the current density can increase further to reach the high levels observed in the device. 
By analyzing the device efficiency and the current density in the HyLEDs with and without the $\mathrm{MoO}_{3}$ injection layer it was possible to estimate the minimum current associated with the minority carriers. Figure 6 shows that the efficacy of the HyLED device with the $\mathrm{MoO}_{3}$ was $0.6 \mathrm{~cd} \mathrm{~A}^{-1}$, which relates to an external quantum efficiency of $0.2 \%$. Taking into account the reported photoluminescence efficiency of $74 \%$ for F8BT and assuming a singlet/triplet ratio and an outcoupling factor both of 1/4, we can estimate that approximately $4 \%$ of the carriers flowing through the device results in exciton formation. ${ }^{17}$ The first thing this tells us is that al least $4 \%$ of the current density must be due to the minority carrier (electrons) that account for a current density of $400 \mathrm{~A} \mathrm{~m}^{-2}$ at $8 \mathrm{~V}$. This is a striking difference from the value observed for the HyLED device in which no $\mathrm{MoO}_{3}$ charge injection layer was used. In that device the current density was only $0.01 \mathrm{~A} \mathrm{~m}^{-2}$ at $8 \mathrm{~V}$. Thus, by altering the injection contact of only one electrode we are able to significantly enhance the injection of the minority carrier (from the unmodified contact). This is in accordance with the simple picture described in Figure 7 and corroborates our previous estimation that in these devices the current is predominantly a hole current.

The second conclusion that can be drawn from the approximation of the minority carrier current density is that a significant number of charges reach the counter electrode prior to recombination, indicating that the $\mathrm{TiO}_{2}$ is not a completely blocking contact for holes. Alternatively, if they recombined, the excitons were generated close to the metal-oxide interface, which efficiently quenched them.

To prevent recombination close to the interface we attempted to decrease the number of holes reaching the interface by adding hole traps to the F8BT layer. $N, N^{\prime}$-diphenyl- $N, N^{\prime}$-bis(3-methylphenyl)-(1, $1^{\prime}$ biphenyl)-4,4'-diamine (TPD) has a HOMO approximately $0.4 \mathrm{eV}$ above the HOMO of the F8BT and can thus act as a hole trap in this matrix. ${ }^{18}{ }^{19}$ Different amounts of TPD were added to a series of devices in an effort to decrease the recombination at the interface. The results shown were obtained from a series of devices prepared on a single batch of $\mathrm{TiO} 2$ substrates.

As can be observed, the current density and luminance of the device with no TPD did not coincide with those depicted in Figures 4 and 5. Figure 8 shows the performance of one series of devices that used a single batch of $\mathrm{TiO}_{2}$ covered substrates. The curves shown in Figures 4 and 5 were obtained using the devices that performed best from a series of approximately 50 prepared devices. The variation in the device performances is attributed to differences between the $\mathrm{TiO}_{2}$ layers.

The addition of TPD dramatically influenced the current level flowing through the device showing that it is an effective charge trap (Fig. 8a). Unfortunately, however, the reduction in the current density was accompanied by a dramatic attenuation in the light output (Fig. 8b). The strong dependence of the current density on the presence of small amounts of hole traps is a strong indicator that the current that flowed through the HyLED device is indeed dominated by holes. This corroborates the simple model in which we suggest that the accumulation of holes at the $\mathrm{F} 8 \mathrm{BT} / \mathrm{TiO}_{2}$ interface is the key factor required to achieve electron injection into the F8BT layer. Thus, an increase in the hole trap density results in a redistribution of the free carriers through the F8BT film modifying the spatial field across the $\mathrm{TiO}_{2} / \mathrm{F} 8 \mathrm{BT}$ interface. ${ }^{20}$ Because of the decreased spatial field, fewer electrons are injected into the F8BT layer and hence the electron emission decreases. Thus, although the addition of TPD resulted in a decrease in current density it decreased the amount of electrons being injected, which also resulted in a significantly lowered luminance. 

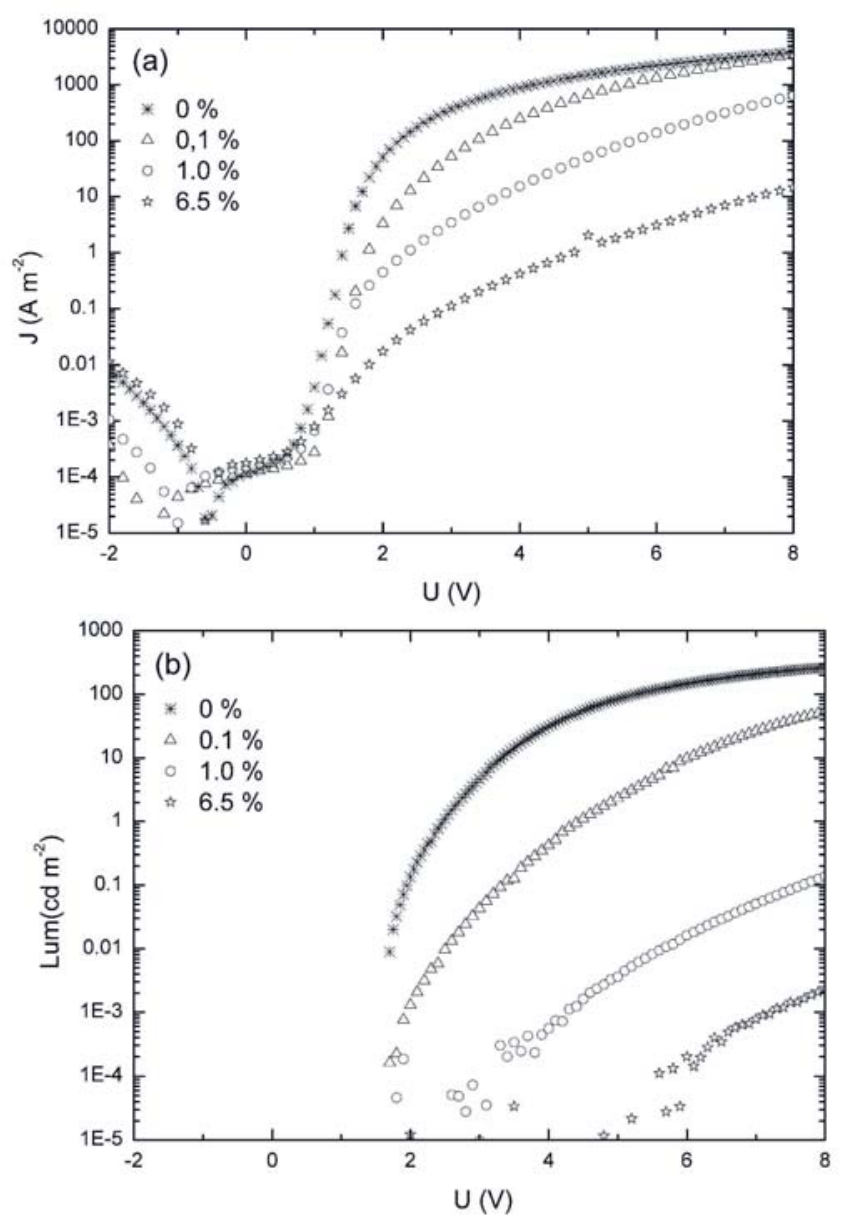

Figure 8. a) Current density and b) luminance versus applied bias for an $\mathrm{ITO} / \mathrm{TiO}_{2} / \mathrm{F} 8 \mathrm{BT} / \mathrm{MoO}_{3} / \mathrm{Au}$ device in forward bias ( ITO biased negatively) with increasing TPD contents (indicated as mass percentage of TPD in F8BT ww \%).

Therefore the device efficacy decreased with increasing amounts of TPD. A possibly better approach consists of introducing a thin hole blocking layer in between the $\mathrm{TiO} 2$ and the F8BT layers. If such a layer is sufficiently thin it may prevent the escape of holes towards the $\mathrm{TiO} 2$ without preventing electron tunneling to the F8BT. This approach is the topic of further optimization studies in our laboratories.

In the above pictured model, it should even be possible to inject electrons from a bare ITO electrode as in this case also an interfacial field will be built up by the holes that are slowly transported through the F8BT layer.

The current density and the luminance for a device in which the ITO is used as the cathode (thus in the HyLED configuration but without the $\mathrm{TiO}_{2}$ interfacial layer) is shown in Figure 9. Surprisingly high current and luminance values are observed considering the energetic gap of at least $1.3 \mathrm{eV}$ between the workfunction of the ITO and the LUMO of the F8BT. This observation confirms our simple model of the operation of the HyLED devices. 


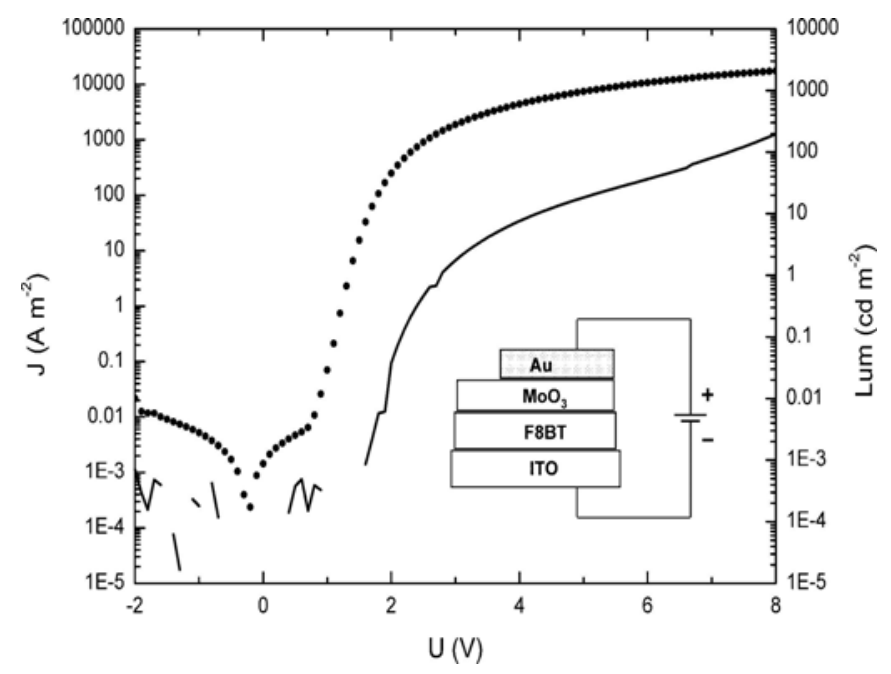

Figure 9. Current density (symbols) and luminance (line) vs. applied bias for an ITO/F8BT/MoO $/ \mathrm{Au}_{\mathrm{device}}$. Inset shows the device layout.

\section{Conclusions}

We have prepared a novel class of hybrid organic-inorganicmaterial- based light-emitting diodes, HyLEDs, making use of the inorganic semiconductor $\mathrm{TiO}_{2}$ and the organic lightemitting polyfluorene F8BT. High brightness levels up to $5700 \mathrm{~cd} \mathrm{~m}^{-2}$ at $8 \mathrm{~V}$ were obtained for devices in which a $\mathrm{MoO}_{3}$ charge injection layer was introduced between the light-emitting polymer and the gold anode. A simple device model consistent with all experimental data is proposed in which the injection of electrons from the metal oxide into the light-emitting layer requires the generation of an interfacial field over the $\mathrm{TiO}_{2}: \mathrm{F} 8 \mathrm{BT}$ interface. In the HyLED described, this interfacial field is generated by the accumulation of holes at that interface. This model implies that the majority carriers responsible for the high current densities are holes.

The device efficacy is low because of the high current density that originates either from insufficient blocking of holes at the $\mathrm{TiO}_{2}$ cathode or from the fact that excitons generated at the interface do not decay radiatively. An attempt to decrease the hole dominated current by the addition of hole traps resulted, apart from the reduction in current density, in a decrease in light emission, confirming that the build up of the interfacial field is a primary requirement for the injection of electrons into the F8BT emitting layer. The strong decrease in the current density upon addition of hole traps provides compelling evidence for a hole dominated current.

Following this simple model, we demonstrated that electroluminescence could even be observed from a device without $\mathrm{TiO}_{2}$ that uses ITO as the cathode. In this device, brightness levels of $200 \mathrm{~cd} \mathrm{~m}^{-2}$ were observed for voltages as low as $8 \mathrm{~V}$, showing that the large barrier for electron injection can be overcome by the interfacial field generated by the accumulation of holes in the light-emitting-polymer layer.

\section{Experimental}

All materials used in this work were obtained either from Aldrich, American Dye Source (F8BT) or from HC Starck (PEDOT:PSS). The metal-oxide layers were prepared using spray pyrolysis using a method described previously. ${ }^{11}$ Briefly, an ethanolic solution of di-iso-propoxy titanium bis(acetyl acetonate) was sprayed, with argon gas, onto a hot ITO substrate $\left(400{ }^{\circ} \mathrm{C}\right)$ and finally annealed at $520{ }^{\circ} \mathrm{C}$ for $2 \mathrm{~h}$. The quality of the films was checked using both SEM (Hitachi S-4800) and AFM (Multimode SPM, Veeco, USA). HyLEDs were prepared by spin coating a thin layer $(50-150 \mathrm{~nm})$ of a light-emitting polymer (LEP) with a chlorobenzene solution. Before spin coating the solutions were filtered using a $0.20 \mu \mathrm{m}$ PTFE filter. After spin coating the thin films were dried and transferred into a high-vacuum chamber, that 
was integrated in an inert atmosphere $\left(<0.1\right.$ ppm $\mathrm{O}_{2}$ and $\left.\mathrm{H}_{2} \mathrm{O}\right)$ glovebox. Gold and $\mathrm{MoO}_{3}$ were thermally evaporated using a base pressure of $1 \times 10^{-6}$ mbar. This served as the anode contact and as an optical mirror to enhance the unidirectional illumination of the device. Reference OLED devices were prepared by spin coating a $100 \mathrm{~nm}$ layer of PEDOT: PSS from an aqueous dispersion. Subsequently deposition of the LEP from a chlorobenzene solution was followed by thermal evaporation of a $\mathrm{Ba} / \mathrm{Ag}$ cathode (5 and $80 \mathrm{~nm}$, respectively). The thickness of the spin coated films was determined using an Ambios XP1 profilometer. $J-V$ characteristics were measured using either a Keithley 2400 source measurement unit or an AutoLab PGSTAT30 potentiostat. Electroluminescence was detected using a Si-photodiode coupled to a Keithley 6485 picoamperometer. The photocurrent was calibrated using a Minolta LS100 luminance meter. Electroluminescent spectra were recorded using an Avantis fiber optics photospectrometer.

\footnotetext{
${ }^{1}$ Y. Sun, N. C. Giebink, H. Kanno, B. Ma, M. E. Thompson, S. R. Forrest, Nature 2006, 440, 908.

${ }^{2}$ T. W. Lee, J. Zaumseil, S. H. Kim, J. W. P. Hsu, Adv. Mater. 2004, 16, 2040.

${ }^{3}$ H. J. Bolink, L. Cappelli, E. Coronado, M. Graetzel, E. Ortí, R. D. Costa, M. Viruela, M. K. Nazeeruddin, J. Am. Chem. Soc. 2006, $128,14786$.

${ }^{4}$ J. D. Slinker, J. Rivnay, J. S. Moskowitz, J. B. Parker, S. Bernhard, H. D. Abruña, G. G. Malliaras, J. Mater. Chem. 2007, 17, 2976

${ }^{5}$ Y. Athanassov, F. P. Rotzinger, P. Péchy, M. Graetzel, J. Phys. Chem. B. 1997, 101, 2558.

${ }^{6}$ Z. Zhang, Z. Deng, C. Liang, M. Zhang, D. Xu, Displays 2003, 24, 231.

${ }^{7}$ K. Morii, M. Ishida, T. Takashima, T. Shimoda, Q. Wang, M. K. Nazeeruddin, M. Graetzel, Appl. Phys. Lett. 2006, 89, 183510.

${ }^{8}$ S. A. Haque, S. Koops, N. Tokmoldin, J. R. Durrant, J. Huang, D. D. C. Bradley, E. Palomares, Adv. Mater. $2007,19,683$.

${ }^{9}$ R. Könenkamp, R. C. Word, M. Godinez, Nanotechnology 2006, 17, 1858.

${ }^{10}$ A. J. Campbell, D. C. Bradley, H. Antoniadis, Appl. Phys. Lett. 2001, 79, 2133.

${ }^{11}$ L. Kavan, M. Graetzel, Electrochim. Acta. 1995, 40, 643.

${ }^{12}$ I. Horcas, R. Fernandez, J. M. Gomez-Rodriguez, J. Colchero, J. Gomez- Herrero, A. M. Baro, Rev. Sci. Instrum. 2007, 78, 013705 .

${ }^{13}$ G. L. Frey, J. R. Reynolds, R. H. Friend, Adv. Mater. 2002, 14, 265.

${ }^{14}$ C. Chu, S. Li, C. Chen, V. Shrotriya, Y. Yang, Appl. Phys. Lett. 2005, 87, 193508.

${ }^{15}$ T. Matsumoto, T. Nakada, J. Endo, M. K., N. Kawamura, A. Yokoi, J. Kido, SID Int. Symp. Dig. Tech. Pap. $2003,979$.

${ }^{16}$ A. J. Campbell, D. D. C. Bradley, H. Antoniadis, Appl. Phys. Lett. 2001, 79, 2133.

${ }^{17}$ K. Murata, S. Cinà, N. C. Greenham, Appl. Phys. Lett. 2001, 79, 1193

${ }^{18}$ D. M. Pai, J. F. Yanus, M. Stolka, J. Phys. Chem. 1984, 88, 4714.

${ }^{19}$ P. M. Borsenberger, D. S. Weis, Organic Photoreceptors for Xerography, Marcel Dekker, New York, 1998

${ }^{20}$ P. W. M. Blom, M. C. J. M. Vissenberg, Phys. Rev. Lett. 1998, 80, 3819
} 